at work in denudation and deposition, the above factors may be verified and allowed for in making an estimate of the antiquity of sediments.

The estimate here given is probably too low in the light of these corrective factors, and it is interesting to notice how much more closely it agrees with the results of the totally independent method based on radio-activity than do those deduced from the facts of sedimentation in the usual way.

Mosuril, Portuguese East Africa, May 6. ARthur Holmes.

\section{Breath Figures.}

THE two interesting letters on breath figures by Lord Rayleigh and Dr. Aitken (Nature, May 25 and June 15) seem to me to contain a statement of the cause of this phenomenon as well as the data necessary to support it.

Thus it is shown that a blow-pipe flame, burning sulphur, sulphuric acid, hydrofluoric acid, and caustic soda give these breath figures, while heat and alcohol flame give no such result. The conclusion apparent from these chemical data is that when the glass is coated with a film having an affinity for water, breath figures are formed.

Coal gas contains sulphur, and a blow-pipe flame gives sufficient sulphuric acid to form a film on glass; burning sulphur gives similar acid products, and both yield breath figures.

Sulphuric acid, hydrofluoric acid, and caustic soda are each capable of dissolving glass, which implies wetting and a certain amount of penetration; washing does not immediately remove this, and a film of acid or alkali is left capable likewise of forming breath figures.

In ammonia solution we have a strong alkali which cannot dissolve glass in the caustic soda sense; when it is allowed even to stand on a glass plate no breath figure is formed, but when it is well rubbed in a faint figure is produced.

If breath figures, from blow-pipe flames, say, be soaked in ammonia solution and washed, they may be gradually destroyed-by neutralisation of the acid in the superficial pores of the glass-until breath outlines only exist. These lines correspond to the lines of greatest acid penetration, and would be represented by charred lines on a piece of wood.

This gradual destruction of the figures on gradual neutralisation of the acid conclusively shows that these figures are neither due to cleanliness nor dust, as has been suggested.

This explanation enables one to predict that Dr. Aitken's suggested experiment of burning pure hydrogen in dustless air would give breath figures, while pure (dusty) hydrogen burning in pure (dusty) oxygen would give no figures, the reasons being that pure hydrogen burning in air gives sufficient nitric acid to produce figures, while pure hydrogen burning in pure oxygen produces no acid. and would produce no figures.

Caeteris paribus, it may be inferred that pure quartz glass would not give figures with sulphuric acid, but with hydrofluoric acid and caustic soda.

If the rays from radium can produce breath figures on glass, it constitutes another cause.

Glasgow.

George Craig.

\section{A Zenith Halo.}

WiLL you permit me to quarrel with your correspondent for the heading "A Zenith Rainbow," attached to his letter from Bruges, published in NATURE of May II, p. 349? The phenomenon described was not a rainbow, as $\mathrm{Mr}$. Gold has taken pains to point out. The heading is unfortunate, for two reasons: first, because it tends to confirm the prevalent misuse of the word "rainbow," and, secondly, because it will probably lead to the improper classification of Mr. Kreyer's letter in bibliographies.

The terminology of atmospheric optics is in a state of dire confusion, even among scientific men, but all the latter are agreed in calling the phenomenon in question a halo. Mr. Gold follows Pernter and most other writers NO. 2175 , VOL. 87 ] in terming this particular halo an " arc of contact." However, this name, as well as the common alternative, "tangent arc," is objectionable, for the reason that the halo thus designated is by no means always in contact with, or tangent to, the halo of $46^{\circ}$ (or the position which the latter would occupy if present). On this subject see M. Besson's article "Le halo du 2 I décembre IgIo; un arc tangent qui n'est pas tangent," in La Nature of March II, I9II, p. 248. In the picture that accompanies M. Besson's article, the "tangent" arc is shown separated from the halo of $46^{\circ}$ by an interval of about $3^{\circ}$.

Another common name, " circumzenithal arc," is open to the objection that this halo is but one of many that are central at the zenith.

The only accurate and distinctive name for the phenomenon is " upper quasi-tangent arc of the halo of $46^{\circ}$."

Statistics of the frequency of the various halo phenomena are misleading. Mr. Gold states, on the authority of Pernter, that the arc in question had been observed only about seventy times up to $188_{3}$. Besson, "Sur la théorie des halos," records III observations of it in ten years (1898-1907) at Montsouris alone. If systematic observations of halos were made all over the world, the frequency of such phenomena would doubtless be found to be far greater than is now generally supposed.

C. Fitzhugh Talmax.

U.S. Weather Bureau, Washington, May 22.

Probably no one will be inclined to dispute $\mathrm{Mr}$ Talman's proposition that systematic observations would largely increase the apparent frequency of the phenomenon mentioned.

With reference to the terminology, it is, as he points out, unfortunate that the terms " arc of contact," "tangent arc," should have come into general use for a bow which is not always in contact with the halo. I cannot, however, agree that Mascart's term, "quasi-tangent arc," is a satisfactory substitute. It was, I believe, intended to meet those cases when the arc is present at approximately $46^{\circ}$ from the sun, but without the $46^{\circ}$ halo. It does not fit cases for low or high solar altitudes when the arc is more than $46^{\circ}$ from the sun. I think it would be better, instead of trying to indicate all the peculiarities of the phenomenon by its name, to use a term such as " auxiliary arc," if the present names are to be abandoned.

The phenomenon is described by Bravais as "un veritable arc-en-ciel," and this may account for the less appropriate use of the term "rainbow."

Meteorological Office, South Kensington,

E. Gold. London, S.W., June 2.

\section{Jelly Rain.}

ON the morning of Saturday, June 24, the ground here was found to be covered with small masses of jelly about as large as a pea. There had been heavy rain on Friday night, and it was raining at 7 a.m., when, so far as 1 can ascertain, the phenomenon was first seen. On being examined microscopically the lumps of jelly turned out to contain numerous ova of some insect, with an advanced embryo in each. The egg itself is very minute-an elongated oval $0.04 \mathrm{~mm}$. in length. Yesterday and the day before many larvæ emerged, and were obviously those of some species of Chironomus, though colourless, having no hæmoglobin, as is the case with the larvæ of $C$. plumosus. Not being an entomologist, I am at a loss to understand how these egg-masses could have appeared where they did unless they were conveyed by the rain, as it does not seem likely that the midges would have laid their eggs on pavements, gravel paths, tombstones, \&c., even had they been wet; nor has any large number of adult insects been seen in the locality. It would be interesting to hear whether the same thing was observed elsewhere, and whether the phenomenon often occurs. Showers of algæ, small snails, and even frogs have been recorded from time to time, but I cannot recall a like instance to the above.

Eton, Bucks, June 30 . 